\title{
Hotline update of clinical trials and registries presented at the German Cardiac Society Meeting 2009
}

\author{
L. S. Maier $\cdot$ S. H. Schirmer $\cdot$ K. Walenta $\cdot$ \\ C. Jacobshagen $\cdot$ M. Böhm
}

Received: 24 April 2009/Accepted: 28 April 2009/Published online: 26 May 2009

(C) Springer-Verlag 2009

\begin{abstract}
This review article gives an overview on a number of novel clinical trials and registries in the field of cardiovascular medicine. Key presentations made at the 75th annual meeting of the German Cardiac Society, held in Mannheim, Germany, in April 2009 are reported. The data were presented by leading experts in the field with relevant positions in the trials and registries. These comprehensive summaries should provide the readers with the most recent data on diagnostic and therapeutic developments in cardiovascular medicine similar as previously reported (Rosenkranz et al. in Clin Res Cardiol 96:457468, 9; Maier et al. in Clin Res Cardiol 97:356-363, 3).
\end{abstract}

Keywords EuroCMR - FITT-STEMI - IRIS · GERSHWIN · REGION · SBK KardioPro - OMEGA · DHR · Stem Cell · Heinz-Nixdorf Recall Study · HIT $\cdot$ DYSIS $\cdot$ FIX-CHF-5 - SYNTAX

\section{EuroCMR}

Cardiovascular magnetic resonance (CMR) has a broad range of clinical applications and is increasingly used in daily clinical practice throughout Europe. Besides left

L. S. Maier · C. Jacobshagen

Abt. Kardiologie \& Pneumologie/Herzzentrum,

Georg-August-Universität Göttingen, Robert-Koch-Str. 40,

37075 Göttingen, Germany

S. H. Schirmer · K. Walenta · M. Böhm ( $\square)$

Universitätsklinikum des Saarlandes, Klinik für Innere Medizin

III, Kardiologie, Angiologie und Internistische Intensivmedizin,

Kirrberger Str., 66421 Homburg, Germany

e-mail: michael.boehm@uniklinikum-saarland.de ventricular function, ischemia as well as myocardial texture can be investigated. During its German pilot phase the EuroCMR registry sought to evaluate indications, image quality, safety and impact on patient management of CMR imaging in clinical routine in a large number of cases. EuroCMR is a multicenter registry in which patients were consecutively enrolled in 29 German CMR centers using web based online case record forms. More than 10,000 patients were included in this ongoing registry between June 2007 and January 2009. Image quality was found to be good in $98 \%$ of cases. Severe complications occurred in a minority of patients $(<1 \%)$ and were all associated with stress testing (using dobutamine or adenosine). However, no patient died during or due to the CMR procedure. The most often used reason to perform a CMR imaging (Fig. 1) was myocarditis/cardiomyopathy (32\%) followed by suspected coronary heart disease or ischemia $(31 \%)$, and myocardial viability $(15 \%)$. Case reading and reporting were mostly done by cardiologists $(>65 \%)$. In more than half of the patients included $(\sim 60 \%)$ CMR findings resulted in a change of patient management. Importantly, in $16 \%$ of cases, the final diagnosis based on CMR was different to the diagnosis before CMR, leading to a complete change in patient management. In $45 \%$ of the cases, no further coronary angiography was needed after CMR. In summary, CMR is frequently performed, seems safe, and provides an important diagnostic tool with high image quality leading to a great clinical impact. (Presenter: O. Bruder, Essen)

\section{FITT-STEMI}

The purpose of the multicenter trial FITT-STEMI (Feedback Intervention and Treatment Times in ST-Elevation 


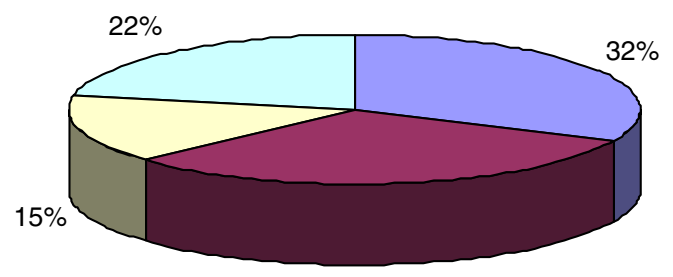

$31 \%$

$\square$ Myocarditis/Cardiomyopathy $\square$ Myocardial Viability
$\square$ suspected Coronary Heart $\square$ Other
Disease/Ischemia

Fig. 1 The most often used reason to perform a CMR imaging

Myocardial Infarction) is to examine if formalized data assessment and systematic feedback improves treatment times (i.e., contact-to-balloon time and door-to-balloon time) and survival rates in patients with myocardial infarction with ST-segment elevation (STEMI) [1]. For many patients with STEMI, the time from presentation to percutaneous coronary intervention (PCI) exceeds established goals $(<120 \mathrm{~min}$ first medial contact-to-balloon time). Till date, only registry data exist suggesting 26 more deaths/1,000 patients for every $60 \mathrm{~min}$ that a PCI is delayed (vs. 90 min contact-to-balloon time) in patients with myocardial infarction. Formalized data feedback is one strategy proposed to reduce treatment time in STEMIpatients. The multicenter trial includes hospitals with primary PCI capacity (with currently two university clinics and four additional hospitals). Existing protocols encourage prompt transfer of patients with STEMI to the PCI center and emphasize minimizing time to treatment. In each participating center, all patients presenting with STEMI are enrolled (intention to treat). The study is conducted prospectively during five consecutive 3-month periods (quarters). Data collection is web-based and identical for the five quarters. For each center, the time points from initial contact with the medical system to revascularization are assessed, analyzed, and presented in an interactive session to hospital and emergency services staff. This formalized data feedback is performed at the end of each quarter. Patients presenting during the first 3-month period are included as the reference group. Data from patients with STEMI presenting during the next four quarters are presented in the same manner. Having included more than 1,200 patients into the study, median contact-to-balloon time could be reduced from 129 to $105 \mathrm{~min}$ and door-to-balloon time from 70 to $50 \mathrm{~min}$ after 1 year. Further analysis will show whether mortality can also be decreased, especially in those patients with high TIMI risk scores of 3-4 and especially $\geq 5$ (Fig. 2). Since this is an ongoing trial, other hospitals may participate additionally in the near future. (Presenter: K. H. Scholz, Hildesheim)

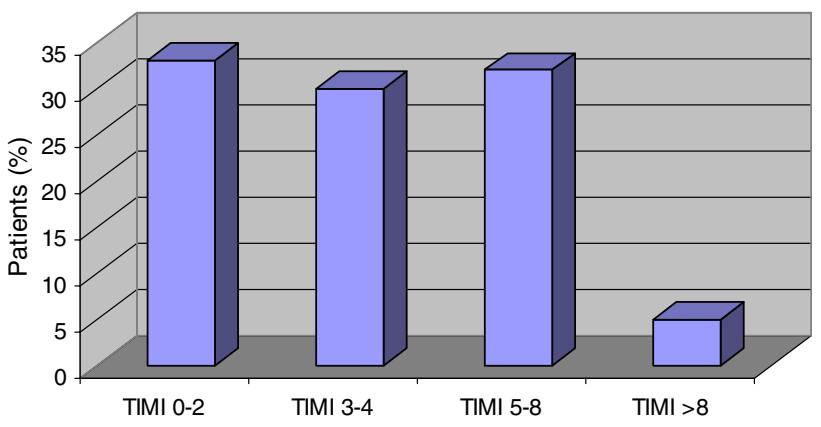

Fig. 2 TIMI risk scores in patients included in FITT-STEMI

\section{IRIS}

Implantable cardioverter defibrillators (ICD) have been shown to be effective for primary prevention of sudden cardiac death in selected groups of patients in the chronic phase after myocardial infarction. The IRIS (Immediate Risk-Stratification Improves Survival) study investigated whether sudden cardiac death could be prevented in high risk patients ( $\mathrm{EF} \leq 40 \%$ and heart rate $\geq 90 / \mathrm{min}$ or nonsustained ventricular tachycardias (VTs) with heart rates $\geq 150 / \mathrm{min}$ ) by ICD implantation $<30$ days after myocardial infarction as compared to optimal medication alone. Primary endpoint was death of all cause. 902 patients were randomized between June 1999 and October 2007 . $76 \%$ of the patients were male, average EF was $34 \%$. Optimal medication consisted of antiplatelet therapy (99\%), $\beta$-blockers (90\%), ACE-inhibitors (82\%), and statins. With respect to the primary endpoint, there was no difference between both the groups over 72 months. Therefore, the authors concluded that there is no evidence that immediate ( $<30$ days) ICD implantation after myocardial infarction improves mortality. (Presenter: D. Andresen, Berlin)

\section{GERSHWIN}

Percutaneous coronary intervention using sirolimuseluting stents (SES) has been shown to reduce instent-restenosis compared to bare metal stents (BMS) [2]. The objective of the GERSHWIN (German stent health outcome and economics within normal practice) study was to study clinical benefit (MACE: death, myocardial infarction, bypass therapy, re-PCI in the target vessel), costs, and quality of life using SES or BMS [3]. Patients were allocated in a 2:1 fashion to SES or BMS from 2003 to 2005 in 35 centers across Germany. Cost calculation was based on estimation of direct costs, including the costs for stent implantation, rehabilitation, re-hospitalization, physician care, medications and other therapy, and 
indirect costs involving disablement. Three-year followup data were available for a total of 274 and 639 patients treated with BMS or SES, respectively. After 3 years, rePCI in the target vessel was significantly reduced from 23.3 to $16.6 \%$ in the SES compared to the BMS group (relative risk reduction of $29 \%$ ), while all other MACE, as well as survival until first MACE, did not differ significantly. As reported earlier, initial hospitalization costs were 2,000 $€$ lower in the BMS group, which was mainly driven by stent costs. Direct follow-up costs were not different between the groups, but indirect follow-up costs were significantly lower in the SES group (1,315 vs. $2,317 €)$. Total costs were slightly higher in the SES group (17,494 vs. $16,295 €)$, resulting in $23,872 €$ for each MACE to be avoided. Quality of life, which has been significantly better 3,6 , and 12 months after SES implantation, was comparable between the two groups 36 months after implantation. In summary, SES leads to significantly less target vessel-revascularization procedures compared to BMS. Total costs were still higher in SES, whereas a decrease in follow-up costs did not compensate for higher initial costs due to stent costs. (Presenter: S. N. Willich, Berlin)

\section{REGION: Regional differences in cardiac mortality in Germany-results of a scientific evaluation}

From 1998 until 2007, more than 2.3 million German death records were manually encoded using WHO ICD10 classification to compare regional patterns of cardiac mortality. Importantly, patient age was standardized according to the European standard population of 1976. Death from ischemic heart diseases (ICD-10 I20-I25), consisting largely of acute myocardial infarction (I20) and chronic ischemic heart disease (I25) showed strong regional variation with high figures $(>155$ cases per 100,000 men) in the north-east and the Saarland, and $<100$ cases in the south, particularly in the south-west of Germany. Figures for woman were generally only $50 \%$ of those of men, with similar regional differences. Regional variation did not change significantly over time (from 1998 to 2007) in men or women. However, numbers of cardiac deaths decreased from 200 per 100,000 men in 1998 to 130 in 2007, and 100 per 100,000 women in 1998 to 70 in 2007. As a limitation, manual coding of the death records is acknowledged. In summary, a clear geographic gradient is found in cardiac mortality, with a reduction in mortality of time. The data can serve for further causal analyses. All-cause mortality was similarly distributed, indicating socioeconomic factors to play a causal role in the regional variation. (Presenter: S. N. Willich, Berlin)

\section{SBK KardioPro}

KardioPro (efficiently facing progression of coronary artery disease by active risk management) is a prevention study hypothesizing that early cardiovascular risk stratification (according to the PROCAM score) and optimal risk factor management delay the onset of coronary artery disease (CAD) and prevent severe courses of the disease. Healthy insurance policy holders $>45$ years are subjected to risk factor assessment and subsequent diagnostic tests depending on the individual risk. In case of moderate or high risk scores, non-invasive stress-testing is performed. If CAD is suspected, the patients are subjected to coronary angiography. Risk assessment is thoroughly discussed with the participants, and individual advice particularly focusing on life style changes is provided. Importantly, modern cardiac diagnostic tools such as CT-angiography, perfusion-MRT or stress-echocardiography have to be available at the participating institutions. All participant visits are documented electronically to build up a large coronary prevention database. Until April 2009, 12,163 participants at more than 80 centers have been included, of whom $70 \%$ have been graded low risk, while CAD was diagnosed in $12 \%$. Most of the participants (73\%) were included into the study in a preventive track, while $20 \%$ were suspected of CAD and $7 \%$ had known CAD. CT-angiography was particularly used in cases where risk factor assessment was inconclusive. Coronary angiography was finally performed in high risk situations including pathological non-invasive tests. Evaluation of KardioPro with respect to clinical endpoints will start in 2009. (Presenter: J. Brachmann, Coburg)

\section{OMEGA}

Omega-3 fatty acids after myocardial infarction reduced mortality in the GISSI-Prevenzione trial [4]. In the OMEGA trial (randomized trial of omega- 3 fatty acids on top of modern therapy after acute myocardial infarction), 3,851 patients were treated with $1 \mathrm{~g}$ omega- 3 fatty acids or placebo from 3 to 14 days after myocardial infarction (STEMI or NSTEMI) for 1 year in addition to optimal medical therapy. In the course of the study, high-risk patients ( $>70$ years old, $\mathrm{EF}<40 \%$, diabetics, lack of revascularization) were selectively included, resulting in a total of $24 \%$ of patients with an EF $<40 \%$. Around $90 \%$ of all study patients were on optimal medical therapy following MI (aspirin, statin, betablocker, ACE-inhibitor). The primary endpoint, sudden cardiac death, occurred in $1.5 \%$ of the patients in both the treatment and the placebo group. Similarly, the secondary endpoints (major adverse cardiac events and arrhythmic events, revascularization, 
Fig. 3 Decrease in hospital mortality in STEMI (a) and STEMI complicated by cardiogenic shock (b)

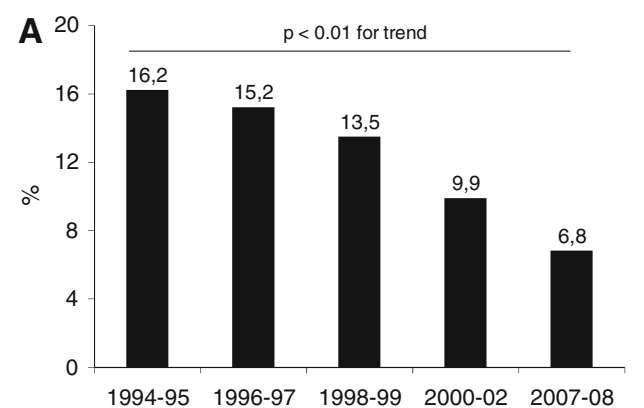

rehospitalization) did not differ between the groups. Serum-triglycerides were significantly decreased in the treatment group (121 vs. $127 \mathrm{mg} / \mathrm{dl})$. In summary, in moderate to high-risk patients, strict guideline treatment is associated with a very low risk of sudden cardiac death $(1.5 \%)$, which could not be further reduced by treatment with omega-3 fatty acids. In contrast to the GISSI-Prevenzione trial, acute revascularization, clopidogrel, statin and GPIIb/IIIa-antagonist treatment were more common in the OMGA trial, which may explain the different findings of the two studies. (Presenter: J. Senges, Ludwigshafen)

\section{DHR}

Of 1,082 German hospitals primarily treating ST-elevation myocardial infarction (STEMI), 280 (26\%) joined the DHR (Deutsches Herzinfarkt Register) registry that aimed at mirroring daily practice of MI treatment in Germany without pre-selecting large or catheter laboratory equipped institutions. More than $80 \%$ of all patients were revascularized by primary PCI, only $5 \%$ by lysis therapy, while $10 \%$ of MI patients did not receive reperfusion therapy, mostly because of the initial ECG was not interpreted as acute STEMI or because of co-morbidities. In hospitals with catheterization laboratory, the primary PCI was performed in $90 \%$ and lysis in only $2 \%$. Hospitals without catheterization facilities performed lysis in $12 \%$ and transferred for primary PCI in $70 \%$. First medical contact to balloon (i.e., revascularization by $\mathrm{PCI}$ ), the time was $200 \mathrm{~min}$ if the patient was first transferred to a hospital without catheterization laboratory and $105 \mathrm{~min}$ if transferred to a center with catheterization facility. Fast treatment of patients with MI decreased hospital mortality from $16.2 \%$ in 1994 to $6.8 \%$ in 2008 (Fig. 3a). At discharge, more than $90 \%$ of the patients were treated according to current guidelines, receiving aspirin, clopidogrel, statin, betablocker, and ACE inhibitor. During the last 15 years, the percentage of primary PCI in cardiogenic shock has strongly risen from $16 \%$ in 1995 to almost $90 \%$ in 2008, reducing mortality from 72 to $38 \%$ (Fig. 3b). In summary, the DHR shows a high percentage of primary PCI in myocardial infarction, a good adherence to the guidelines concerning optimal medical therapy post MI, a low inhospital mortality but still high mortality in patients presenting with cardiogenic shock. (Presenter: U. Zeymer, Ludwigshafen)

\section{Stem cells in AMI}

In the past years, several studies have shown that intracoronary administration of bone marrow-derived progenitor cells may have a beneficial effect in cardiovascular disease [5]. Improved left ventricular function in patients at risk for adverse remodeling after acute myocardial infarction has been correlated with stem cell application. All these studies had in common that recanalization therapy and stem cell application were performed within the first hours during myocardial infarction. In this presented randomized, double-blind, placebo-controlled trial, a total of 42 patients with myocardial infarction have been enrolled at least $6 \mathrm{~h}$ after recanalization therapy. Only patients with a creatine kinase $>1,000 \mathrm{U} / 1$ were included. 29 of these patients received bone-marrow-derived stem cells $\left(381 \pm 130 \times 10^{6}\right.$ mononuclear cells $)$ and 13 received placebo. By means of contrast-enhanced magnetic resonance imaging (MRI), left ventricular volumes and $\mathrm{EF}$ were determined at baseline and compared at 1, 3 and 6 months of follow-up. At all three investigated points of time, no significant improvement $(P=0.80)$ of the $\mathrm{EF}$, diameters of the left ventricle during diastole (LVEDD) and systole (LVESD) or the size of myocardial scar tissue could be observed. As these results lacked benefit, this led to the immediate stop of this study. (Presenter: J. Wöhrle, Ulm)

\section{Heinz-Nixdorf Recall study}

First serious cardiac events are often fatal, and half of all CHD deaths are sudden coronary deaths and occur out of the hospital. Quantification of subclinical atherosclerosis has been suggested to improve cardiovascular risk stratification 
beyond established risk factor assessment [6]. The prospective cohort-study evaluated 4,137 patients $(53 \%$ women) without known coronary artery disease regarding predictors and signs of subclinical atherosclerosis. The patients were aged between 45 and 75 years and were investigated from December 2000 until August 2003. The area of coronary artery calcification (CAC) was measured by Electronbeam tomography. CAC was defined as at least four contiguous pixels with a CT density $\geq 130$ Hounsfield Units. The patient cohort was divided into three groups corresponding to the ATP III category (low, intermediate and high risk). The 5-year outcome data showed that the CAC-score is a better predictor of cardiovascular disease compared to classical risk factors such as smoking, hypertension, diabetes or obesity. However, the risk prediction was more valid in men than in women and is suggested to improve risk prediction of patients, especially with intermediate risk. Determination of CAC can be used for reclassification of persons with moderate stroke risk (10-20\%) Beyond this, patients with low-risk having a 10-year-risk below $10 \%$ have no benefit of this risk stratification. (Presenter: R. Erbel, Essen)

\section{HIT}

HIT (Herz im Takt) is a new concept based on a contract between the university hospital of Schleswig-Holstein and surgeries (cardiologists) improving medical treatment of patients with coronary artery disease (CAD). The goal of all partners of this contract was to improve the quality of life of all patients with CAD and to prevent heart attacks. The established cardiologist performed the first outpatient diagnosis and risk classification, and then transferred the patient to the university hospital where coronary angiography could be performed. Afterwards, the patient was discharged and continued treatment in care of his cardiologist. Through close coordination and interaction between outpatient, inpatient and rehabilitative treatment double examinations could be avoided and the patient had an optimal quality of care offered. In consequence of this close cooperation, a higher rate of intake of the prescribed medication was shown which could be attributed to the studyrelated compliance. Betablockers were shown to be taken in $76 \%$ of all cases after 1 year versus $65 \%$ at the beginning. The next aim is to establish this contract-concept throughout Germany. (Presenter: E. Schuhr, Hamburg)

\section{DYSIS}

The main known risk factors enhancing development and progression of cardiovascular diseases are lifestyle,

\begin{tabular}{|c|c|c|c|c|c|c|}
\hline & All & High risk & CVD & $\begin{array}{l}\text { Diabetes } \\
\text { w'o CVD }\end{array}$ & $\begin{array}{l}\text { Score } \\
\geq 5 \%\end{array}$ & $\begin{array}{c}\text { Score } \\
<5 \%\end{array}$ \\
\hline & $n=21, \pi 91$ & $n=17 S E 3$ & $n=10,587$ & $n=4,324$ & $n=2,472$ & $n=4,214$ \\
\hline TC $n \alpha$ at god [3] & 54.4 & 52.1 & 46.5 & 51.9 & 76.0 & 63.9 \\
\hline LDL-C not at goal [\&] & 48.5 & 46.8 & 41.9 & 45.3 & 707 & 55.8 \\
\hline LOW HDL-C [\%] & 26.4 & 28.3 & 30.6 & 29.9 & 15.2 & 18.7 \\
\hline Elevaled TG & 38.8 & 39.6 & 38.5 & 44.5 & 35.5 & 35.3 \\
\hline
\end{tabular}

Fig. 4 Persistent lipid disorders with ongoing statin therapy in correlation with the risk of cardiovascular complications

obesity, hypertension, diabetes [7] and lipid disorders. Large randomized studies have proved in the past, the effectiveness of lipid lowering by statins. Since then, they belong to standard therapy in the secondary and primary prevention of cardiovascular disease. Nevertheless, many patients do not reach the recommended lipid values [8]. DYSIS (Dyslipidemia International Study) is an international, prospective register-study evaluating the prevalence of persistent lipid disorders despite current medical therapy with statins. From April 2008 to February 2009, a total of 22,063 patients with already ongoing statin therapy were enclosed via 2,987 surgeries in 11 European countries and Canada. Nearly half of the patients undergoing chronic statin therapy did not reach the target levels for LDLcholesterol as recommended in the guidelines (Fig. 4). Additionally, two-thirds of these patients had further abnormal values for HDL-cholesterol or triglycerides. The results of DYSIS demonstrate the discrepancy between recommendations of professional societies and clinical practice in the treatment of lipid disorders in terms of primary and secondary prevention, and the potential for improvements in medical treatment of the outpatient belonging to the risk groups. (Presenter: A. K. Gitt, Ludwigshafen)

\section{US multicenter RCT of cardiac contractility modulation in patients with advanced heart failure (FIX-CHF-5)}

Congestive heart failure (CHF) remains one of the most frequent causes of morbidity and mortality. Despite major advances in pharmacological therapy and/or device-based treatment, millions of patients suffer from CHF with heart failure symptoms NYHA III or IV and less than half of these patients are eligible for resynchronization therapy. A new approach with cardiac contractility modulation (CCM) has been investigated in the FIX-CHF-4 study. Here, it was shown that CCM treatment was associated with clinically and statistically significant improvements in 

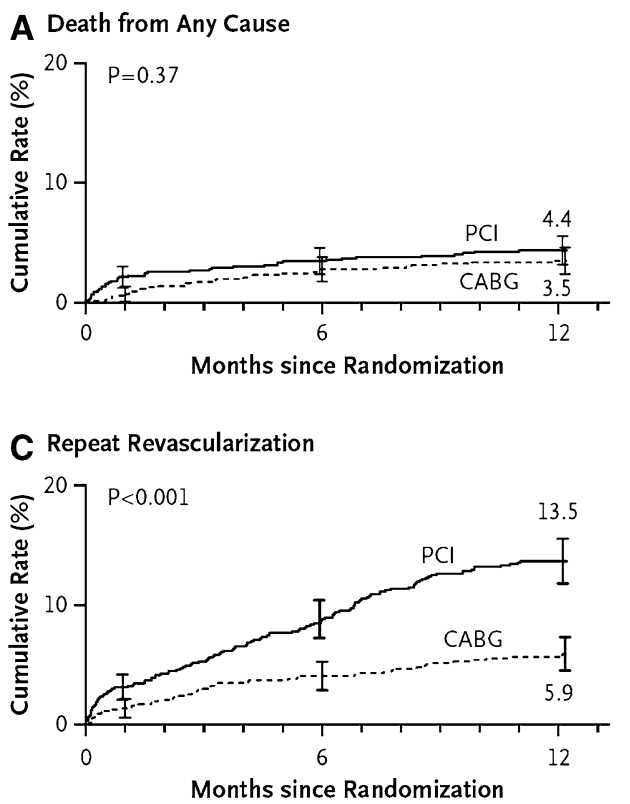

Fig. 5 Rates of outcomes among the study patients, according to treatment group [10]. Kaplan-Meier curves are shown for the percutaneous coronary intervention (PCI) group and the coronaryartery bypass grafting $(\mathrm{CABG})$ group for death from any cause (Panel $A$ ); death, stroke, or myocardial infarction (MI) (Panel B); repeat revascularization (Panel $C$ ); and the composite primary end point of major adverse cardiac or cerebrovascular events (Panel D). The two groups had similar rates of death from any cause (relative risk with PCI vs. CABG, $1.24 ; 95 \%$ confidence interval $[\mathrm{CI}], 0.78-1.98)$ and

exercise tolerance and quality of life [9]. CCM signals are intended to improve the strength of muscle contraction. This new concept based on observations that electrical impulses delivered to the right ventricular septum during the heart's absolute refractory period appear to enhance left ventricular contractility without actually causing excitation. The FIX-CHF-5 is a pivotal trial underway at 50 sites in the United States. The FIX-CHF-5 study was a multicenter, prospective, double-blind, crossover study conducted in 428 patients with NYHA class III or IV [ejection fraction $(\mathrm{EF}) \leq 35 \%$ ] despite optimal therapy. All patients had QRS complexes within normal limits and patients with atrial fibrillation were excluded. 95\% of patients had an implantable cardioverter-defibrillator (ICD). Primary end point was the anaerobic threshold (AT). Co-secondary endpoints concernded exercise tolerance (peak $V_{2}$ ) and quality of life. In the study design, all patients received a device that was switched off at time of implantation to ensure double blinding. After a 2-week run-in period, 215 patients had their device switched on, whereas $213 \mathrm{kept}$ it switched-off and received only medical treatment. Nonexcitatory impulses to the heart were delivered $5 \mathrm{~h}$ per day. The safety endpoint of mortality and hospitalization after 12 months was not different in both groups. Moreover, the primary efficacy endpoint (anaerobic threshold) could not
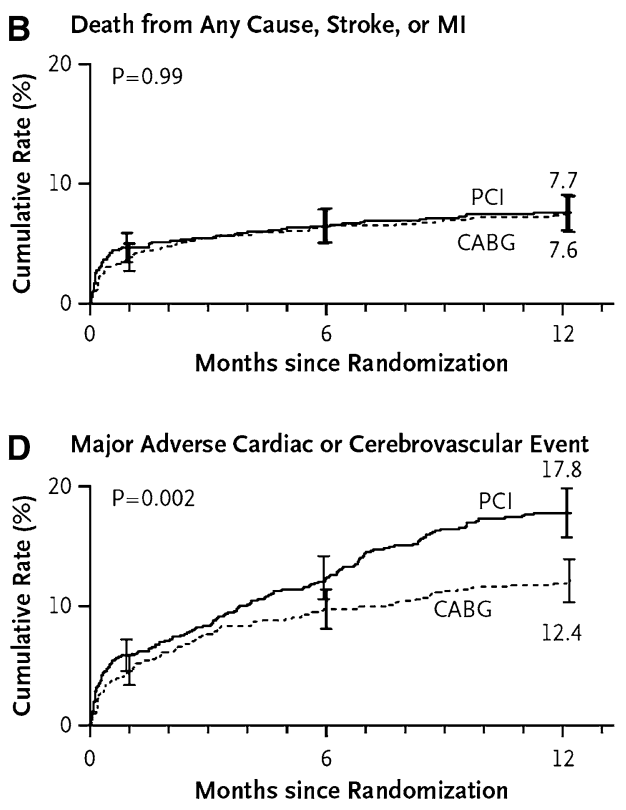

rates of death from any cause, stroke, or MI (relative risk with PCI vs. CABG, $1.00 ; 95 \% \mathrm{CI}, 0.72-1.38)$. In contrast, the rate of repeat revascularization was significantly increased with PCI (relative risk, $2.29 ; 95 \% \mathrm{CI}, 1.67-3.14)$, as was the overall rate of major adverse cardiac or cerebrovascular events (relative risk, 1.44; 95\% CI, 1.151.81). The I bars indicate 1.5 SE. Relative risks were calculated from the binary rates. $P$ values were calculated with the use of the chisquare test

be reached by most of the investigated patients and therefore could not be really taken into account in the results. However, quality of life, NYHA classification and $V_{2}$ significantly $(P<0.001, \quad P=0.0026$ and $P<0.024)$ increased in patients with CCM compared to medical treatment alone. Besides these findings, a subgroup analysis revealed a significant benefit regarding the primary endpoint of AT in patients with an EF $\leq 25 \%$ with NYHA III $(P=0.04)$ and NYHA IV $(P=0.19)$. (Presenter: M. Borggrefe, Mannheim)

\section{SYNTAX}

The SYNTAX (Synergy between percutaneous coronary intervention with TAXUS and cardiac surgery) trial was an international, multicenter, randomized, controlled comparison of coronary-artery bypass grafting (CABG) versus PCI with drug-eluting stents in 1,800 patients with previously untreated three-vessel or left main coronary artery disease (or both) [10]. The primary clinical end point was a composite of major adverse cardiac and cerebrovascular events (i.e., death from any cause, stroke, myocardial infarction, or repeat revascularization). A noninferiority comparison of the two groups demonstrated significant 
higher rates of major adverse cardiac or cerebrovascular events at 12 months in the PCI group (17.8 vs. $12.4 \%$ for CABG, $P<0.001)$. This result was driven by an increased rate of repeat revascularization in the PCI group (13.5 vs. $5.9 \%$ for CABG, $P<0.001)$. In an analysis of secondary endpoints, the two groups had similar rates of death from any cause or myocardial infarction and of the combined end point of death from any cause, stroke, or myocardial infarction (7.6\% for PCI and $7.7 \%$ for CABG). The 12 month rates of symptomatic graft occlusion (CABG group) and stent thrombosis (PCI group) were similar $(P<0.89)$. Stroke was significantly more likely to occur with CABG ( 2.2 vs. $0.6 \%$ with PCI, $P<0.003$ ). The rate of major adverse cardiac or cerebrovascular events among patients with left main coronary artery disease was similar in the CABG and PCI groups (13.7 and 15.8\%, respectively, $P<0.44$, see Fig. 5). To predict outcomes related to anatomical characteristics, the SYNTAX score was designed. Patients with low or intermediate SYNTAX scores in the CABG and in the PCI group had similar rates of major adverse cardiac or cerebrovascular events, whereas among patients with high scores (indicating more complex coronary anatomy), the event rate was significantly increased in the PCI group. Notable strengths and limitations and clinical implications of the SYNTAX trial were discussed by the presenters. Strengths: it was a prospective, multicenter trial in which a large number of patients were enrolled at 85 centers in Europe and the United States. It attempted to include "all comers" with left main or three-vessel coronary artery disease (or both) with an impressively high enrollment rate $(59 \%$ of all screened, eligible patients were included). The study used state-of-the-art CABG and PCI (with arterial grafts and drug-eluting stents, respectively). Limitations: the followup period was only 12 months; the outcomes of PCI and CABG over a longer period of follow up in patients with severe coronary artery disease are unknown. The primary (composite) endpoint was driven by the revascularization rate. With respect to hard endpoints (death from any cause or myocardial infarction and the combined end point of death from any cause, stroke, or myocardial infarction), there was no significant difference between the two groups. But these endpoints were secondary endpoints with observational character. All discussants agreed that in patients with left main or three-vessel coronary artery disease, all pertinent data, including that from diagnostic angiography, should be reviewed by a team consisting of a cardiac surgeon, and an interventional cardiologist to determine the likelihood of safe and effective revascularization with PCI and CABG. The SYNTAX score, indicating the complexity of the coronary anatomy, identifies patients with increased risk of a suboptimal outcome with PCI. (Presenters: T. Meinertz, Hamburg; F. W. Mohr, Leipzig; G. C. Schuler, Leipzig).

Acknowledgments M. Böhm [9] and L.S. Maier [3, 9] are funded by the Deutsche Forschungsgemeinschaft (DFG, Klinische Forschergruppe (KFO 115 [3], Maier; KFO 196 [9], Böhm), HeisenbergProgram [3]).

\section{References}

1. Scholz KH, Hilgers R, Ahlersmann D, Duwald H, Nitsche R, von Knobelsdorff G, Volger B, Möller K, Keating FK (2008) Contactto-balloon time and door-to-balloon time after initiation of a formalized data feedback in patients with acute ST-elevation myocardial infarction. Am J Cardiol 101:46-52

2. Zellerhoff C, Schneider S, Senges J, Pfannebecker T, Hamm C, Tebbe U (2008) Sirolimus-eluting stents in the treatment of chronic total coronary occlusions: results from the prospective multi-center German Cypher Stent Registry. Clin Res Cardiol 97:253-259

3. Maier LS, Maack C, Ritter O, Böhm M (2008) Hotline Update of Clinical Trials and Registries presented at the German Cardiac Society Meeting 2008: PEPCAD, LokalTax, INH, German Ablation Registry, German Device Registry, DES.DE Registry, DHR, Reality, SWEETHEART Registry, ADMA, GERSHWIN. Clin Res Cardiol 97:356-363

4. Rupp H (2006) Omega-3 fatty acids in secondary prevention after myocardial infarct. Clin Res Cardiol 95(Suppl 6):VI12-VI16

5. Bartsch T, Brehm M, Zeus T, Kögler G, Wernet P, Strauer BE (2007) Transplantation of autologous mononuclear bone marrow stem cells in patients with peripheral arterial disease (the TAMPAD study). Clin Res Cardiol 96:891-899

6. Bauer M, Möhlenkamp S, Lehmann N, Schmermund A, Roggenbuck U, Moebus S, Stang A, Mann K, Jöckel KH, Erbel R (2009) The effect of age and risk factors on coronary and carotid artery atherosclerotic burden in males-Results of the Heinz Nixdorf Recall Study. Atherosclerosis. doi:10.1016./j.atherosclerosis.2009. 01.005

7. Bakris G, Böhm M, Dagenais G, Diener HC, Fujita T, Gorelick P, Kjeldsen SE, Laakso M, Mancia G, Pitt B, Sharma A, Sleight P, Teo K, Unger T, Weber M, Williams B, Zannad F (2008) Cardiovascular protection for all individuals at high risk: evidencebased best practice. Clin Res Cardiol 97:713-725

8. Lankisch M, Füth R, Gülker H, Lapp H, Bufe A, Haastert B, Martin S, Rathmann W (2008) Screening for undiagnosed diabetes in patients with acute myocardial infarction. Clin Res Cardiol 97:753-759

9. Rosenkranz S, Maier LS, Maack C, Böhm M (2007) Hotline Update of Clinical Trials and Registries presented at the German Cardiac Society Meeting 2007: 2L-Registry, Kardio-Pro, EVER, AFFECT, VTACH, ARTS II, OPTAMI, PEPCAD I, PEPCAD II, GERSHWIN, SPICE, FIX-CHF and CREDIT. Clin Res Cardiol 96:457-468

10. Serruys PW, Morice MC, Kappetein AP, Colombo A, Holmes DR, Mack MJ, Ståhle E, Feldman TE, van den Brand M, Bass EJ, Van Dyck N, Leadley K, Dawkins KD, Mohr FW (2009) SYNTAX Investigators. Percutaneous coronary intervention versus coronary-artery bypass grafting for severe coronary artery disease. N Engl J Med 360:961-972 